\title{
Liquid crystalline and antinematic behavior of shape-persistent macrocycles from molecular-dynamics simulations
}

\author{
Carlos Avendaño and Erich A. Müller* \\ Department of Chemical Engineering, Imperial College London, South Kensington Campus, London SW7 2AZ, United Kingdom
}

(Received 28 May 2009; published 28 December 2009)

\begin{abstract}
In this work we present a molecular-dynamics study of a coarse-grained (CG) model for a system of planar shape-persistent macrocycles (SPMs). SPMs are synthetic organic rigid macromolecules typically comprised of meta- and para-aromatics groups connected by acetylene and/or diacetylene units. In the CG model, each SPM is represented as a rigid hexagonal arrangement of 24 soft-repulsive spheres, resembling a large ring or hoop. The supramolecular arrangement of these macrocycles at high pressures is studied using $N-P-T$ moleculardynamics simulation both by expansion of an initial hexagonal lattice structure and also by compression of an isotropic phase. In both cases, systems under consideration exhibit an isotropic-smectic- $A$ phase transition, which is detected by monitoring relevant order parameters and analyzing snapshots of equilibrium configurations. The smectic- $A$ phase is unique; although the molecules form layers, the system presents antinematic order where the orientation of the molecular axes is perpendicular to the direction of the layers themselves. Due to their planar geometry, the SPM molecules would be expected to form columnar or nematic phases. On the contrary, these phases seem suppressed by a novel smectic- $A$ phase, formed by the mutual interpenetration of the cycles. These results are a unique example of how molecular nonconvexity can, by itself, induce mesomorphism in anisotropic systems.
\end{abstract}

DOI: 10.1103/PhysRevE.80.061702

PACS number(s): 61.30.-v, 61.20.Ja

\section{INTRODUCTION}

It was shown by Onsager that the stability of liquidcrystalline (LC) phases in systems comprised of athermal anisotropic particles, e.g., disk-shaped and rod-shaped particles interacting with hard-core repulsive interactions, may be explained as a competition between orientational entropy, which favors the isotropic state, and the entropy associated with minimizing excluded volume, which favors the ordered nematic phase. [1] Over the years, simulation and theory aimed at attempting to elucidate the properties of anisotropic particles have mainly been concentrated on systems of hard rodlike particles [2-15], leaving hard discotic mesogenes in a secondary role. This trend is presumably due to the larger number of applications of the elongated systems. However, discotic liquid crystals are gaining interest for their potential optical and electronic applications.

Typically, molecular discotic mesogens are synthesized by arranging aromatic rings to form flat structures which are surrounded by flexible alkyl chains. [16-19] An example was first reported by Chandrasekhar and co-workers in 1977; they showed that a family of hexaesters of benzene molecules can form LC phase in which molecules are stacked to form a columnar phase, mainly driven by $\pi-\pi$ interactions between aromatic cores [20].

In systems of hard disks, typically isotropic $(I)$, nematic $(N)$, and columnar (Col) LC phases have been observed by computer simulation [21-23], by experiments on molecular discogens [16-19], and in suspensions of colloidal platelets [24-26]. In the case of the Col phase, different types of mesophases can be found depending on the two-dimensional

\footnotetext{
*Corresponding author; e.muller@imperial.ac.uk
}

(2D) symmetry in which the columns are organized [16]. Different models of hard discotic molecules have been studied using molecular simulation including thin hard disks $[21,23,27]$, hard cut spheres [22], hard cylinders [28], hard tetragonal parallelepipeds [29], discotic hard spherocylinders [30], oblate spheroids [31-33], and Gay-Berne particles [34] among others.

Recently, a new family of discotic liquid crystals, called shape-persistent macrocycles (SPMs), has begun to attract attention. SPMs are organic macromolecules synthesized with meta- and para-aromatic groups typically connected by acetylene and diacetylene units in such a way that the final rigid structure cannot collapse $[35,36]$. This persistent shape is different from, for example, the molecular structure of crown ethers where the macrocycles are flexible. The synthesis of SPMs has experienced a boom due to their potential applications in chemistry and materials science. SPMs can be used in different areas such as in molecular recognition, selective complexation, and, more recently, they have been used as large molecular building blocks to assemble new supramolecular compounds [37-40]. In particular, one of the most fascinating macrocycles synthesized is the system reported by Höger and co-workers [36] which is depicted in Fig. 1(a). This system is comprised of a rigid macrocycle, made of ten phenylene subgroups, connected through acetylene units with alkyl chains attached to the backbone. Two primary architectures can be envisioned depending on whether the phenylene group, to which the alkyl chain is directly attached, can or cannot freely rotate in the ring structure. Surprisingly, only when the phenylene group is able to rotate does the system exhibit LC phase behavior-in this case a nematic. This feature is possible only when the lateral flexible chain can fold back into the cavity of its own ring. In this way, the interpenetration of lateral chains of neighboring 


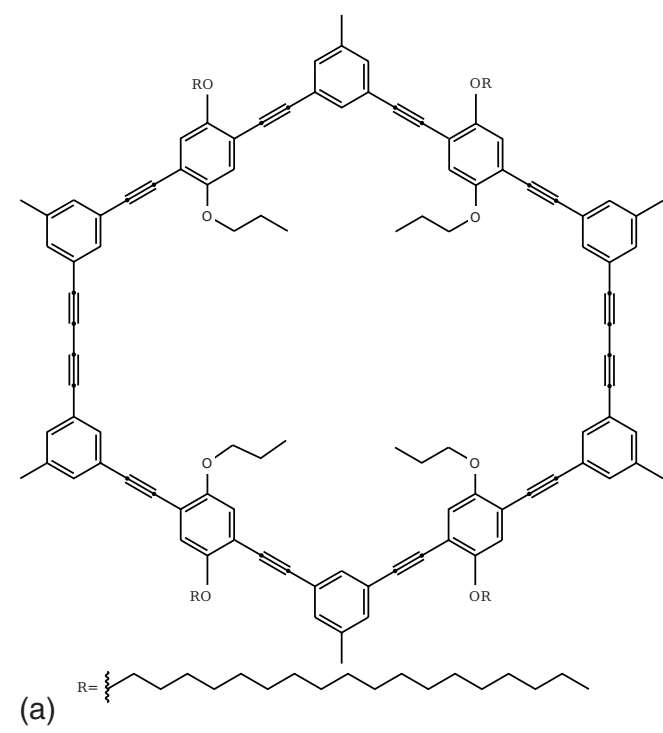

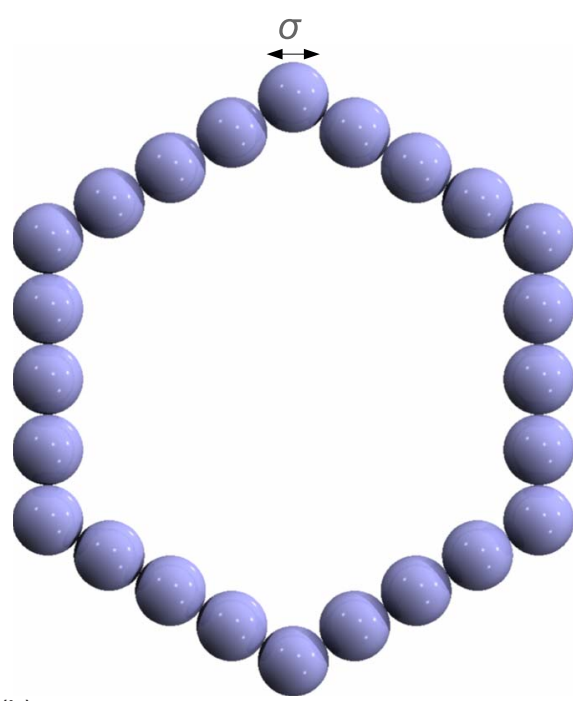

(b)
FIG. 1. (Color online) (a) SPM reported by Höger and co-workers [36], comprising phenylene groups connected through acetylene and diacetylene units; (b) representation of the ungrafted SPM model used in this work. The system is comprised of 24 spheres of diameter $\sigma$ in a hexagonal arrangement to form a rigid unit. The beads interact via a WCA reference potential [50]. In this system, the principal molecular axis, $\hat{\mathbf{u}}$, is pointing out in direction perpendicular to the plane of the ring. particles and the entanglement of different chains with those of other macrocycles are discouraged. Other examples of SMPs with different functional groups have been reported in the literature (see, for example, $[39,41,42]$ ). Furthermore, the synthesis of giant macrocycles, with cavity sizes up to $\sim 60 \AA$, composed of thiophene, acetylene, and ethylene building blocks, has also been reported $[43,44]$.

From the molecular-simulation point of view, these systems have not been studied; the purpose of this work is to redress this lack. Initially, we are interested in determining whether rigid macrocycles, interacting mainly with repulsive interactions, are able to form LC phases. Most common features of LC phases can be explored by analyzing simplified coarse-grained models of molecules where the shape and anisotropy are well represented by a repulsive (or softrepulsive) core. This is the approach taken here. As will be shown, in this system, an isotropic-smectic- $A$ phase transition has been observed. The smectic- $A(\operatorname{Sm} A)$ phase is characterized by the formation of layers where the principal orientation axis of the macrocycles is in the plane of the layers. Furthermore, the computation of the biaxility order parameter gives an indication that there is no secondary preferential order direction in the system. This phenomenon, where particles are orientated perpendicularly to the system director, is often called antinematic order and is characterized by negative value of the nematic order parameter and has been observed only in anisotropic systems subject to external electromagnetic fields [45-49]. It is important to stress that this antinematic behavior in our system is basically due to the nonconvex shape of the molecules. The analogous convex system, i.e., hexagonal-shaped colloidal platelets, has been found to have only an $I-N$ phase transition [23].

The structure of the rest of the paper has been organized as follows. In Sec. II, the model of the macrocycles studied in this work and the details of the molecular simulation are presented. In Sec. III, we present the molecular-dynamics (MD) simulation results obtained for the macrocycles for both expansion and compression of the system. Finally, in Sec. IV, we present the conclusions of the present work.

\section{SIMULATION DETAILS}

The system analyzed in this work consists of rigid macrocycles comprised of 24 beads of diameter $\sigma$ and mass $m$ in a hexagonal arrangement [see Fig. 1(b)]. The particles interact via the WCA reference potential, introduced in the perturbation theory of Weeks, Chandler, and Andersen [50]. This potential is purely repulsive and is given by

$$
u_{\mathrm{WCA}}(r)= \begin{cases}4 \epsilon\left[\left(\frac{\sigma}{r}\right)^{12}-\left(\frac{\sigma}{r}\right)^{6}\right]+\epsilon, & r \leq 2^{1 / 6} \sigma \\ 0, & r>2^{1 / 6} \sigma,\end{cases}
$$

where $r$ is the center-to-center distance between different beads and $\epsilon$ is the well depth of the associated Lennard-Jones potential.

We introduce conventional reduced units to describe the different thermodynamic states and structural properties. Reduced temperature, density, pressure, and time are defined within this work as $T^{*}=k_{B} T / \epsilon, \rho^{*}=N_{b} \sigma^{3} / V, P^{*}=P \sigma^{3} / \epsilon$, and $t^{*}=t\left(\epsilon / m \sigma^{2}\right)^{1 / 2}$, where $T$ is the absolute temperature, $k_{B}$ is the Boltzmann constant, $N_{b}$ is the total number of beads, $V$ is the system volume, $P$ is the pressure, and $t$ is the time. Similarly, all distances are given in units of $\sigma$.

MD simulations of $N_{m}=512$ molecules (corresponding to $N_{b}=12288$ beads) were carried out in the $N-P-T$ ensemble employing a Nosé-Hoover thermostat [51] and a Hoover barostat, using the Melchionna modification [52], in a cubic simulation box of volume $V$ with periodic boundary conditions. For all the simulations, we employed a leapfrog integration algorithm with a time step of $\delta t^{*} \approx 0.004$. The simulations were performed at a temperature of $T^{*}=1.25$ in order to reproduce as closely as possible the properties of the hardsphere model when using the WCA potential $[53,54]$. The number of time steps used in this work to equilibrate the system was $1.5 \times 10^{6}$ and additional $2.0 \times 10^{6}$ time steps were used to obtain averaged values. Near to the phase transition, more time steps were required in order to obtain reliable results. In order to observe the degree of hysteresis in the system, we report both the expansion from a hexagonal 
TABLE I. NPT-MD simulation results for pressure $P^{*}$, number density $\rho^{*}$, order parameters $S$ and $\tau$, smectic layer spacing $d_{l}^{*}$, and type of liquid-crystalline phase obtained from expansion of a hexagonal lattice. $I=$ isotropic and $\operatorname{Sm} A=$ smectic $-A$.

\begin{tabular}{|c|c|c|c|c|c|}
\hline$P^{*}$ & $\rho^{*}$ & $S$ & $\tau$ & $d_{l}$ & Phase \\
\hline 0.297 & $0.336 \pm 0.002$ & $-0.414 \pm 0.002$ & $0.899 \pm 0.012$ & $8.33 \pm 0.02$ & $\operatorname{Sm} A$ \\
\hline 0.282 & $0.333 \pm 0.002$ & $-0.415 \pm 0.003$ & $0.902 \pm 0.013$ & $8.35 \pm 0.02$ & $\operatorname{Sm} A$ \\
\hline 0.266 & $0.328 \pm 0.002$ & $-0.418 \pm 0.003$ & $0.907 \pm 0.013$ & $8.39 \pm 0.02$ & $\operatorname{Sm} A$ \\
\hline 0.251 & $0.324 \pm 0.002$ & $-0.417 \pm 0.002$ & $0.903 \pm 0.014$ & $8.43 \pm 0.02$ & $\operatorname{Sm} A$ \\
\hline 0.235 & $0.318 \pm 0.002$ & $-0.422 \pm 0.010$ & $0.900 \pm 0.017$ & $8.48 \pm 0.03$ & $\operatorname{Sm} A$ \\
\hline 0.219 & $0.313 \pm 0.002$ & $-0.415 \pm 0.007$ & $0.894 \pm 0.018$ & $8.51 \pm 0.03$ & $\operatorname{Sm} A$ \\
\hline 0.204 & $0.304 \pm 0.002$ & $-0.419 \pm 0.003$ & $0.883 \pm 0.015$ & $8.62 \pm 0.03$ & $\operatorname{Sm} A$ \\
\hline 0.188 & $0.296 \pm 0.002$ & $-0.452 \pm 0.002$ & $0.895 \pm 0.016$ & $8.70 \pm 0.03$ & $\operatorname{Sm} A$ \\
\hline 0.172 & $0.288 \pm 0.002$ & $-0.461 \pm 0.002$ & $0.894 \pm 0.016$ & $8.76 \pm 0.03$ & $\operatorname{Sm} A$ \\
\hline 0.157 & $0.278 \pm 0.002$ & $-0.451 \pm 0.003$ & $0.868 \pm 0.023$ & $8.87 \pm 0.03$ & $\operatorname{Sm} A$ \\
\hline 0.149 & $0.271 \pm 0.002$ & $-0.443 \pm 0.005$ & $0.848 \pm 0.025$ & $8.94 \pm 0.03$ & $\operatorname{Sm} A$ \\
\hline 0.141 & $0.261 \pm 0.002$ & $-0.412 \pm 0.005$ & $0.799 \pm 0.028$ & $9.04 \pm 0.04$ & $\operatorname{Sm} A$ \\
\hline 0.133 & $0.250 \pm 0.002$ & $-0.355 \pm 0.013$ & $0.718 \pm 0.039$ & $9.15 \pm 0.05$ & $\operatorname{Sm} A$ \\
\hline 0.125 & $0.227 \pm 0.002$ & $0.062 \pm 0.023$ & $0.094 \pm 0.098$ & & $I$ \\
\hline 0.117 & $0.220 \pm 0.002$ & $0.060 \pm 0.022$ & $0.063 \pm 0.024$ & & $I$ \\
\hline 0.110 & $0.213 \pm 0.002$ & $0.064 \pm 0.024$ & $0.062 \pm 0.023$ & & $I$ \\
\hline 0.102 & $0.206 \pm 0.002$ & $0.063 \pm 0.024$ & $0.061 \pm 0.022$ & & $I$ \\
\hline 0.094 & $0.198 \pm 0.002$ & $0.060 \pm 0.021$ & $0.060 \pm 0.024$ & & $I$ \\
\hline 0.086 & $0.190 \pm 0.002$ & $0.066 \pm 0.022$ & $0.059 \pm 0.022$ & & $I$ \\
\hline 0.078 & $0.181 \pm 0.002$ & $0.060 \pm 0.022$ & $0.058 \pm 0.022$ & & $I$ \\
\hline 0.070 & $0.172 \pm 0.002$ & $0.062 \pm 0.022$ & $0.057 \pm 0.021$ & & $I$ \\
\hline 0.063 & $0.163 \pm 0.002$ & $0.060 \pm 0.022$ & $0.057 \pm 0.020$ & & $I$ \\
\hline 0.055 & $0.153 \pm 0.002$ & $0.057 \pm 0.022$ & $0.054 \pm 0.019$ & & $I$ \\
\hline 0.047 & $0.142 \pm 0.002$ & $0.054 \pm 0.019$ & $0.056 \pm 0.020$ & & $I$ \\
\hline 0.039 & $0.130 \pm 0.002$ & $0.051 \pm 0.018$ & $0.052 \pm 0.019$ & & $I$ \\
\hline 0.031 & $0.116 \pm 0.002$ & $0.049 \pm 0.019$ & $0.054 \pm 0.019$ & & $I$ \\
\hline 0.023 & $0.101 \pm 0.001$ & $0.046 \pm 0.017$ & $0.053 \pm 0.019$ & & $I$ \\
\hline 0.016 & $0.083 \pm 0.001$ & $0.043 \pm 0.015$ & $0.053 \pm 0.018$ & & $I$ \\
\hline 0.008 & $0.057 \pm 0.001$ & $0.055 \pm 0.023$ & $0.054 \pm 0.018$ & & $I$ \\
\hline
\end{tabular}

lattice ultimately into the isotropic phase and a compression from a low-density isotropic state to high-density states. All MD simulations were performed using the DL_POLY simulation package version 2.20 developed by Smith and coworkers $[55,56]$.

In order to detect possible formation of mesophases, different order parameters may be used. The orientational order can be analyzed from the second-rank tensor $\mathbf{Q}$, defined as $[57,58]$

$$
\mathbf{Q}=\frac{1}{N_{m}} \sum_{j=1}^{N_{m}}\left(\frac{3}{2} \hat{\mathbf{u}}_{j} \otimes \hat{\mathbf{u}}_{j}-\frac{\mathbf{I}}{2}\right),
$$

where $\mathbf{u}_{j}$ is the principal molecular axis (which is perpendicular to the ring, i.e., is oriented out of the plane of the page in Fig. 1) of particle $j, \otimes$ denotes dyadic product, and I is the second-rank unit tensor. Diagonalization of the tensor yields the eigenvalues $\lambda^{+} \geq \lambda^{0} \geq \lambda^{-}$and the corresponding orthonormal eigenvectors $\hat{\mathbf{n}}^{+}, \hat{\mathbf{n}}^{0}$, and $\hat{\mathbf{n}}^{-}$. The nematic order parameter, $S$, is defined as the dominant eigenvalue (i.e., the eigenvalue of greatest absolute value) of $\mathbf{Q}$ and the corresponding eigenvector is the preferred direction for the system (director) [58]. Depending of the sign of $S$, different types of order can be observed $[48,49]$. First, if $0<S \leq 1$, the particles are preferentially aligned along the principal director, corresponding to normal nematic order. On the other hand, if $-0.5 \leq S<0$, the particles are aligned in a plane perpendicular to the principal director. The latter case is often called antinematic order. The formation of a possible second preferential direction in the system (biaxial order) can be identified using the difference $\Delta$ between the two smallest (in absolute value) eigenvalues of the matrix $\mathbf{Q}$. If they are degenerate, all directions perpendicular to the director are equally probable and the system has unaxial symmetry $(\Delta$ $\sim 0$ ).

The possible formation of layers in the system was monitored using the translational order parameter $\tau$, obtained by simulation using the following expression: 
(a)

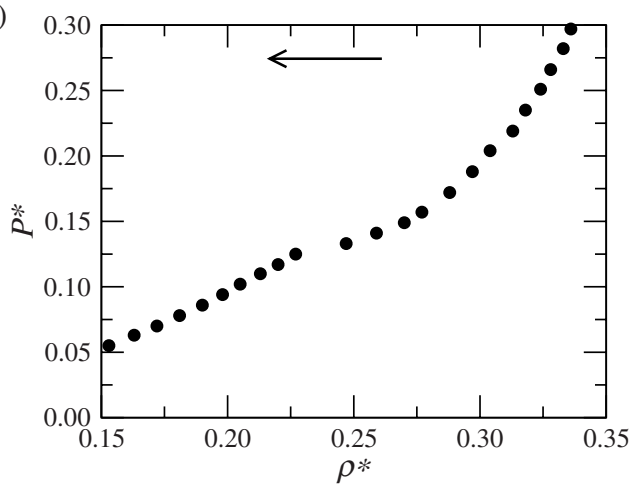

(c)

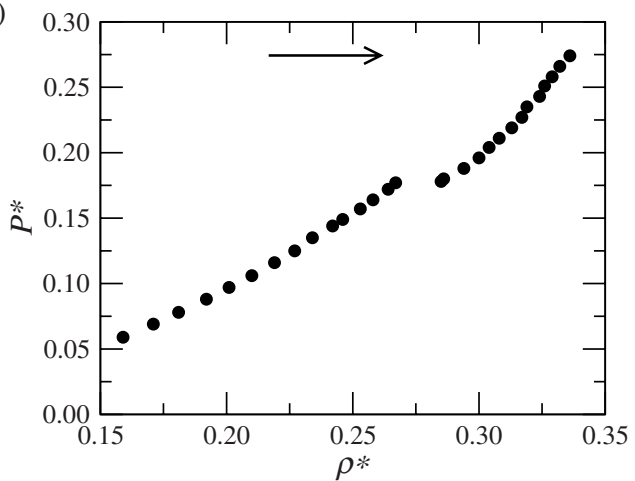

(b)

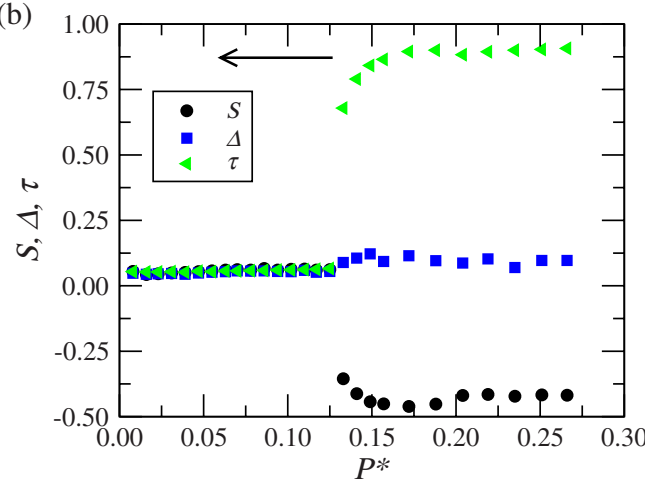

(d)

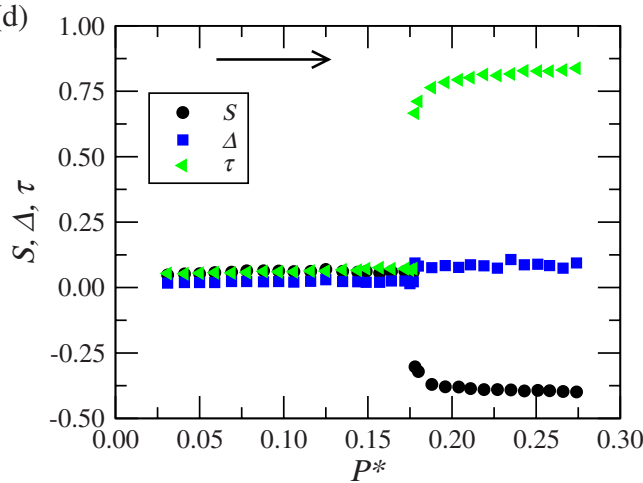

FIG. 2. (Color online) Pressure, $P^{*}$, as a function of the number density, $\rho^{*}$, and the nematic order parameter, $S$, biaxial order parameter, $\Delta$, and translational order parameter, $\tau$, as a function of the pressure $P^{*}$, for a system of 512 macrocycles at temperature $T^{*}=1.25$. (a) and (b) correspond to the expansion of the system from a hexagonal lattice (data not shown) to a smectic phase and further to an isotropic state, while (c) and (d) correspond to the compression from an isotropic state to a smectic state.

$$
\tau(\mathbf{k})=\left\langle\left|\frac{1}{N_{m}} \sum_{j=1}^{N_{m}} \exp \left(i \mathbf{k} \cdot \mathbf{r}_{j}\right)\right|\right\rangle,
$$

where $\mathbf{r}_{j}$ is the position of the center of mass of particle $j$, $\mathbf{k}=2 \pi \hat{\mathbf{l}} / d_{l}$ is the reciprocal-lattice vector, $\hat{\mathbf{l}}$ is the unit vector normal to the plane formed by the layers, and $d_{l}$ is the smectic layer spacing. The parameter $\tau$ corresponds to the amplitude of the smectic layers and it can be seen as the first coefficient in the Fourier expansion of the particle density $[59,60]$. As in the case of the nematic order parameter $S$, the unit vector $\hat{\mathbf{I}}$ and the smectic layer spacing $d_{l}$ are not known a priori. In order to calculate the unit vector $\hat{\mathbf{l}}$, we have used a similar method to that in Ref. [61], where $\hat{\mathbf{l}}$ is obtained as the vector that is maximally orthogonal to the set of molecular orientation vectors. This problem is written as

$$
\min (|\mathbf{U} \mathbf{l}|) \text { subject to }|\mathbf{l}|=1,
$$

where $\mathbf{U}$ is the $N_{m} \times 3$ matrix containing the molecules' orientation $\hat{\mathbf{u}}_{j}$ in its rows. The solution of this problem can be achieved using the singular value decomposition (SVD) method [62]. Once the layer direction $\hat{\mathbf{I}}$ is known, we calculate the periodicity of the layers $d_{l}$. This can be done evaluating Eq. (3) for different trial layer spacing in a convenient range and taking $d_{l}$ as the value that maximizes the expression $[23,60]$.

\section{RESULTS}

As a starting point, an arbitrary initial crystalline hexagonal phase was destabilized, by reducing the pressure in sequential manner, until the $\operatorname{Sm} A$ phase was observed. Between the hexagonal lattice and $\operatorname{Sm} A$ phase, states with nematic ordering were observed. However, in this work, we did not pursue the equilibrium properties of these phases because the $\operatorname{Sm} A$ phase deserves a further description as it has some novel aspects. Furthermore, the nematic states observed during expansion of the lattice may be metastable or fictitious, as they are not observed upon compression of lowdensity isotropic states.

In Table I, the MD simulation results for the pressure $P^{*}$, number density $\rho^{*}$, order parameters $S$ and $\tau$, smectic layer spacing $d_{l}^{*}=d_{l} / \sigma$, and type of LC phase are presented for the case of expansion of the ordered system. The behaviors of the pressure as a function of the number density and that of the order parameters as a function of the number density are shown in Figs. 2(a) and 2(b). Upon expansion, a first-order $I-\operatorname{Sm} A$ phase transition from a smectic state $\left(\rho^{*}=0.247\right.$, $S=-0.355, \tau=0.679)$ to an isotropic state $\left(\rho^{*}=0.227\right.$, $S=0.062, \tau=0.066)$ was observed at $P^{*} \sim 0.129$. This transition is determined by observing discontinuities in the density, and nematic and translational order parameters, as can be observed in Figs. 2(a) and 2(b). Moreover, no evidence of biaxial order has been observed since the biaxial order parameter, $\Delta$, in the smectic phase branch does not present significant values. Negative values of the nematic order pa- 


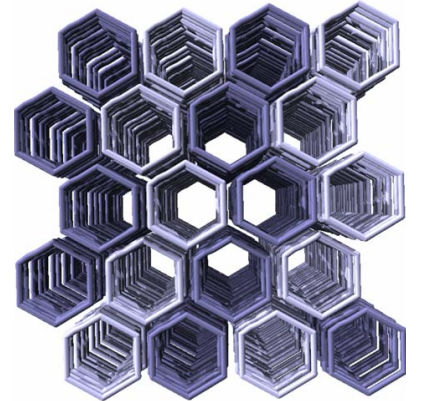

(a)

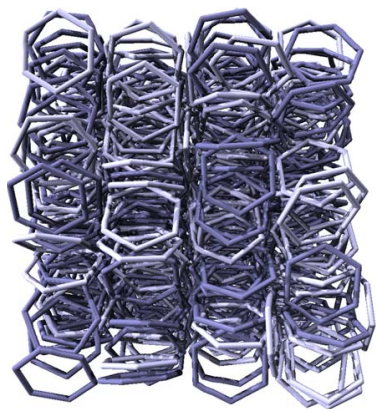

(b)
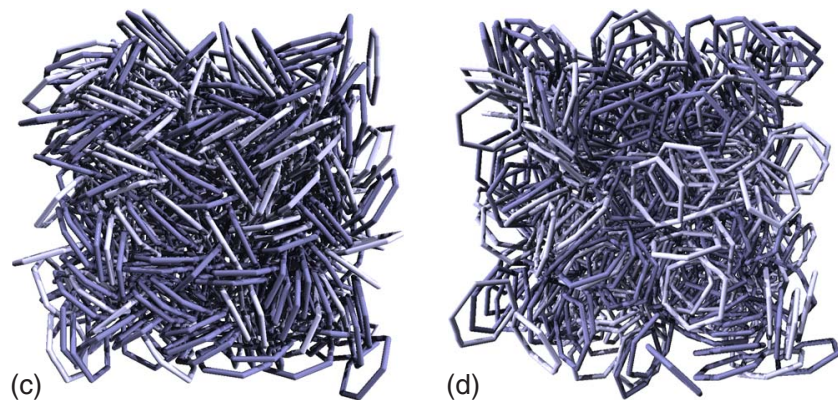

FIG. 3. (Color online) Snapshots for three different configurations for 512 macrocycles at temperature $T^{*}=1.25$ obtained by expansion of the system from a lattice. (a) Initial lattice; (b) $\mathrm{Sm} A$ state at $P^{*}=0.157$ with a side view of the layers; (c) the same state as (b) but viewed perpendicular to the layers (e.g., in the direction of $\hat{\mathbf{l}}$ ); (d) highest-density $I$ state at $P^{*}=0.125$. To aid visualization, a random half of the molecules in each configuration is shaded in different color and cores of the spherical beads are replaced by thick lines of diameter $\sigma / 2$.

rameter, $S$, in the $\operatorname{Sm} A$ phase reveal that the particle axes are mainly oriented in the direction perpendicular to the layer, i.e., the system presents antinematic order.

In Fig. 3, snapshots of the initial lattice configuration, an intermediate $\operatorname{Sm} A$ phase configuration (viewed perpendicular and along the direction of the layers), and the highest-density isotropic state are shown. Although the biaxial order parameter reveals no order in the plane of the layers in the $\operatorname{Sm} A$ phase, in Fig. 3(c), clusters of particles oriented in parallel directions can be observed. The absence of long-range order in the plane of the layers in the $\operatorname{Sm} A$ phase can also be observed in Fig. 4, where the projection of the structure factor in this plane is shown. In this plot, the isotropic rings confirm the absence of order in the layer.

The formation of this $\operatorname{Sm} A$ phase is unexpected as the planar geometry of the system would suggest the appearance of nematic or columnar phases instead of the smectic one. However, it seems that the huge cavity in the ring and the lack of directional interactions (for example, dipolar or quadrupolar interactions between the beads that would foster the alignment and stacking of the rings) allows the particles to interpenetrate each other and fill the cavities of neighboring particles, avoiding in this way the formation of ordered structures in the layers. This behavior is interesting as they showcase how the nonconvex shape of the particles can alter the molecular mechanism of the formation of mesophases. In

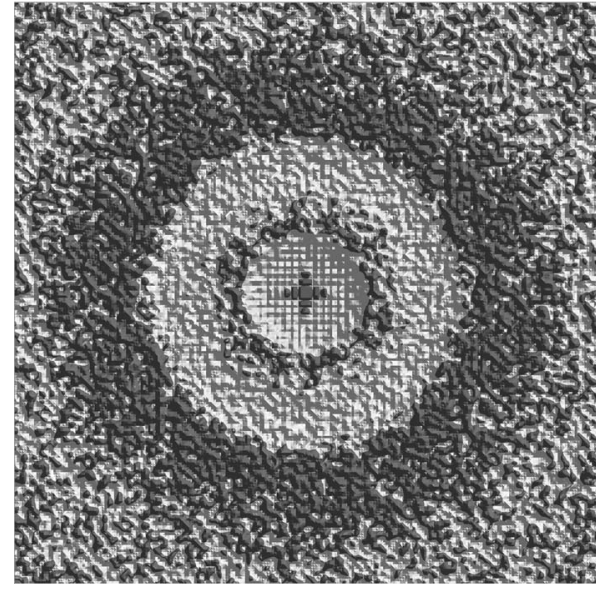

FIG. 4. Projection of the structure factor in the plane of the layers for the system of 512 macrocycles at temperature $T^{*}=1.25$ for the $\operatorname{Sm} A$ phase state at $P^{*}=0.157$ obtained during the expansion of the system from a hexagonal lattice (cf. Fig. 3). Darker shades indicate higher values of the structure factor.

terms of Onsager's excluded-volume arguments [1], one can recognize that if the particles interpenetrate, i.e., one particle can partially occupy some of the free volume within another's annular region, the reduction in total volume in the system induced by the molecular rearrangement would result in a decrease of the free energy and thus in the stability of the resulting phase. This simple argument alone explains the suppression of the columar (nematic) phase in favor of the smectic- $A$ phase. These smectic phases have presumably not been reported before as this is a geometrical requirement with respect to the size of the "hole" required in the otherwise-discotic particle.

A similar destabilization of nematic and columnar phases that leads to an $I-\operatorname{Sm} A$ transition has been reported in the molecular simulation of spherical fan-shaped molecules by Vanakaras and Photinos [63], where molecules are made of three fused infinitely thin disks. The geometry of these fanshaped molecules resembles, in some sense, the structures in our model as the particles interpenetrate each other, thus pre-

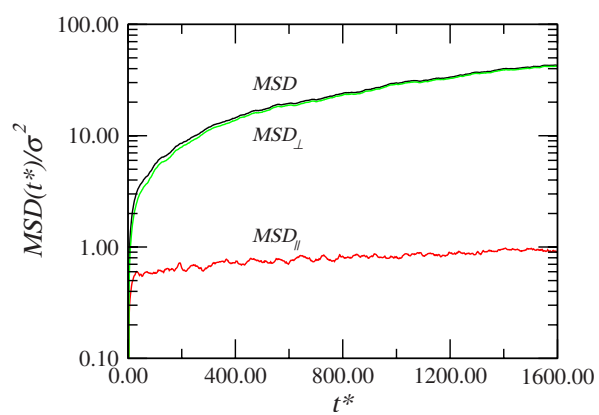

FIG. 5. (Color online) Mean square displacement for the system of 512 macrocycles at temperature $T^{*}=1.25$ for the $\operatorname{Sm} A$ phase state at $P^{*}=0.157$, obtained during the expansion of the system from a hexagonal lattice (cf. Fig. 3). The overall MSD, the mean-square displacement along the plane of the structure $\left(\mathrm{MSD}_{\perp}\right)$, and the mean-square displacement in line with the director, $\hat{\mathbf{l}}$, of the planes $\left(\mathrm{MSD}_{\|}\right)$are shown. 
TABLE II. NPT-MD simulation results for pressure $P^{*}$, number density $\rho^{*}$, order parameters $S$ and $\tau$, smectic layer spacing $d_{l}^{*}$, and type of liquid-crystalline phase obtained from the compression of a low-density isotropic state. $I=$ isotropic and $\operatorname{Sm} A=$ smectic- $A$.

\begin{tabular}{|c|c|c|c|c|c|}
\hline$P^{*}$ & $\rho^{*}$ & $S$ & $\tau$ & $d_{l}$ & Phase \\
\hline 0.031 & $0.116 \pm 0.002$ & $0.048 \pm 0.017$ & $0.054 \pm 0.018$ & & $I$ \\
\hline 0.041 & $0.132 \pm 0.002$ & $0.052 \pm 0.020$ & $0.053 \pm 0.020$ & & $I$ \\
\hline 0.050 & $0.146 \pm 0.002$ & $0.053 \pm 0.021$ & $0.055 \pm 0.020$ & & $I$ \\
\hline 0.059 & $0.159 \pm 0.002$ & $0.057 \pm 0.019$ & $0.057 \pm 0.020$ & & $I$ \\
\hline 0.069 & $0.171 \pm 0.002$ & $0.059 \pm 0.022$ & $0.056 \pm 0.021$ & & $I$ \\
\hline 0.078 & $0.181 \pm 0.002$ & $0.064 \pm 0.023$ & $0.058 \pm 0.021$ & & $I$ \\
\hline 0.088 & $0.192 \pm 0.002$ & $0.064 \pm 0.021$ & $0.062 \pm 0.024$ & & $I$ \\
\hline 0.097 & $0.201 \pm 0.002$ & $0.063 \pm 0.024$ & $0.060 \pm 0.022$ & & $I$ \\
\hline 0.106 & $0.210 \pm 0.002$ & $0.061 \pm 0.021$ & $0.060 \pm 0.022$ & & $I$ \\
\hline 0.116 & $0.219 \pm 0.002$ & $0.062 \pm 0.022$ & $0.063 \pm 0.025$ & & $I$ \\
\hline 0.125 & $0.227 \pm 0.002$ & $0.069 \pm 0.029$ & $0.063 \pm 0.025$ & & $I$ \\
\hline 0.135 & $0.234 \pm 0.002$ & $0.062 \pm 0.020$ & $0.068 \pm 0.026$ & & $I$ \\
\hline 0.144 & $0.242 \pm 0.002$ & $0.059 \pm 0.020$ & $0.066 \pm 0.025$ & & $I$ \\
\hline 0.149 & $0.246 \pm 0.002$ & $0.062 \pm 0.022$ & $0.072 \pm 0.029$ & & $I$ \\
\hline 0.157 & $0.253 \pm 0.002$ & $0.056 \pm 0.018$ & $0.076 \pm 0.035$ & & $I$ \\
\hline 0.164 & $0.258 \pm 0.002$ & $0.061 \pm 0.025$ & $0.072 \pm 0.027$ & & $I$ \\
\hline 0.172 & $0.264 \pm 0.002$ & $0.062 \pm 0.022$ & $0.072 \pm 0.028$ & & $I$ \\
\hline 0.175 & $0.266 \pm 0.002$ & $0.047 \pm 0.015$ & $0.067 \pm 0.026$ & & $I$ \\
\hline 0.177 & $0.267 \pm 0.002$ & $0.059 \pm 0.022$ & $0.072 \pm 0.030$ & & $I$ \\
\hline 0.178 & $0.286 \pm 0.002$ & $-0.303 \pm 0.006$ & $0.666 \pm 0.026$ & $9.68 \pm 0.05$ & $\operatorname{Sm} A$ \\
\hline 0.180 & $0.286 \pm 0.003$ & $-0.321 \pm 0.006$ & $0.711 \pm 0.062$ & $9.38 \pm 0.08$ & $\operatorname{Sm} A$ \\
\hline 0.188 & $0.294 \pm 0.002$ & $-0.370 \pm 0.003$ & $0.764 \pm 0.013$ & $9.41 \pm 0.06$ & $\operatorname{Sm} A$ \\
\hline 0.196 & $0.300 \pm 0.002$ & $-0.379 \pm 0.002$ & $0.784 \pm 0.014$ & $9.21 \pm 0.05$ & $\operatorname{Sm} A$ \\
\hline 0.204 & $0.304 \pm 0.002$ & $-0.380 \pm 0.002$ & $0.794 \pm 0.012$ & $9.15 \pm 0.06$ & $\operatorname{Sm} A$ \\
\hline 0.211 & $0.308 \pm 0.002$ & $-0.386 \pm 0.002$ & $0.802 \pm 0.012$ & $9.16 \pm 0.04$ & $\operatorname{Sm} A$ \\
\hline 0.219 & $0.313 \pm 0.002$ & $-0.390 \pm 0.002$ & $0.814 \pm 0.009$ & $9.13 \pm 0.05$ & $\operatorname{Sm} A$ \\
\hline 0.227 & $0.317 \pm 0.002$ & $-0.390 \pm 0.001$ & $0.810 \pm 0.011$ & $9.06 \pm 0.03$ & $\operatorname{Sm} A$ \\
\hline 0.235 & $0.319 \pm 0.002$ & $-0.391 \pm 0.002$ & $0.816 \pm 0.009$ & $8.94 \pm 0.04$ & $\operatorname{Sm} A$ \\
\hline 0.243 & $0.325 \pm 0.002$ & $-0.395 \pm 0.002$ & $0.828 \pm 0.008$ & $8.97 \pm 0.04$ & $\operatorname{Sm} A$ \\
\hline 0.251 & $0.326 \pm 0.002$ & $-0.393 \pm 0.001$ & $0.827 \pm 0.008$ & $8.95 \pm 0.04$ & $\operatorname{Sm} A$ \\
\hline 0.258 & $0.328 \pm 0.002$ & $-0.394 \pm 0.001$ & $0.827 \pm 0.008$ & $8.87 \pm 0.03$ & $\operatorname{Sm} A$ \\
\hline 0.266 & $0.332 \pm 0.002$ & $-0.397 \pm 0.001$ & $0.831 \pm 0.008$ & $8.85 \pm 0.03$ & $\operatorname{Sm} A$ \\
\hline 0.274 & $0.342 \pm 0.002$ & $-0.399 \pm 0.002$ & $0.845 \pm 0.007$ & $8.78 \pm 0.03$ & $\operatorname{Sm} A$ \\
\hline
\end{tabular}

venting the alignment between neighboring particles. As in our system, in fan-shaped molecules, only an I-SmA phase transition was detected. It is clear that $N$ and $\mathrm{Col}$ phases can be possible only if the SPM particles do not present a large central void or if strong, e.g., hydrogen bonds favor specific configurations. This can also be done, for example, by attaching lateral chains to the backbone which can rotate and fill the cavity, as observed experimentally [36].

In order to test the stability of the high-density states, we have calculated the mobility of the particles. This mobility is monitored using the mean-square displacement (MSD) of the center of mass and the analog $\mathrm{MSD}_{\perp}$ perpendicular to the director $\hat{\mathbf{l}}$, i.e., along the plane of the structure and $\mathrm{MSD}_{\|}$ parallel to the direction of $\hat{\mathbf{l}}$, i.e., across the layers $[64,65]$. In Fig. 5, we report these quantities for the $\operatorname{Sm} A$ phase at $P^{*}$
$=0.157$, corresponding to the same $\operatorname{Sm} A$ state point reported in Fig. 3. As can be observed in Fig. 5, the MSD increases in the planes of the layers while the diffusion of the particles across the layers is low and almost constant as expected for smectic phases. It is apparent that this high-density system is not trapped in dynamic-arrested states.

The compression of the system starting from a lowdensity isotropic state reveals that this system presents some hysteresis, as can be observed in Figs. 2(c) and 2(d). The molecular-simulation results of this compression are reported in Table II. By compression, this system exhibits the same $I-\operatorname{Sm} A$ transition but at higher pressures and densities. This transition going from an isotropic state $\left(\rho^{*}=0.267, S\right.$ $=0.059, \tau=0.072)$ to a smectic $-A$ state $\left(\rho^{*}=0.285, S=\right.$ $-.303, \tau=0.666)$ is observed approximately at the pressure of 


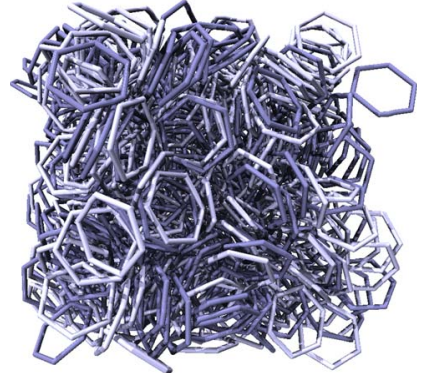

(a)

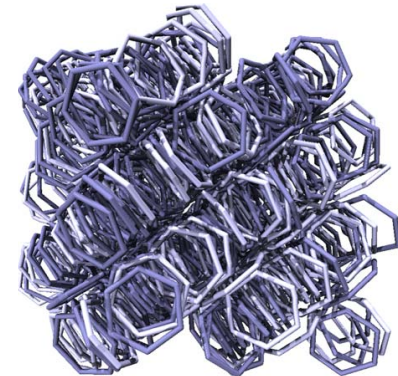

(b)

FIG. 6. (Color online) Snapshots for two different configurations for 512 macrocycles at temperature $T^{*}=1.25$, obtained by compression of the system from an isotropic state. (a) Highestdensity isotropic state at $P^{*}=0.172$; (b) smectic- $A$ state at $P^{*}$ $=0.235$. To aid visualization, particles are drawn in the same fashion as in Fig. 3

$P^{*} \sim 0.178$. The structure of the $\operatorname{Sm} A$ phases obtained by the compression of the system presents the same characteristics observed during the expansion of the system, where mutual interpenetration between molecules in the same layers has been observed. As in the expansion, no biaxial order has been detected. The particles are again oriented perpendicular to the principal system director (antinematic order). It is apparent that the $\operatorname{Sm} A$ phase found is not an artifact for the initial lattice configuration used. The slightly lower values of $\tau$ in the $\operatorname{Sm} A$ phase, compared to the values reported during expansion, imply that the smectic layers are less defined and the final structures show more defects as some of the macrocycles are trapped in between the layers; additionally, the layers are no longer aligned with the simulation-box axes. These are most likely artifacts of the finite size and time of the simulations. Some of these configurations are presented in Fig. 6 where the snapshots of the highest-density $I$ state at $P^{*}=0.177$ and the $\operatorname{Sm} A$ state at $P^{*}=0.266$ are shown.

\section{CONCLUSIONS}

In this work, we have reported an MD simulation study of a model of hard SPMs. This system has been studied using both expansion of a hexagonal lattice and compression from an isotropic state. In both cases, the system exhibits an $I-\operatorname{Sm} A$ phase transition. This $I-\operatorname{Sm} A$ transition is an interesting and rather surprising novel aspect since, in view of the planar geometry of the molecules, one might expect the formation of $N$ or Col phases and not the spontaneous appearance of layers. The $\operatorname{Sm} A$ phase is formed by the arrangement of the macrocycles in the layers with the principal molecular axis perpendicular to the direction of the layers themselves. Moreover, the $\operatorname{Sm} A$ phase does not show biaxial order mainly due to the mutual interpenetration between molecules filling the cavities of neighboring particles thus preventing possible alignment of the molecular axes which would promote the formation of $N$, Col, or possible biaxial phases. Recently, a related mesophase has been observed in the socalled gigantocycles that were synthesized using bananashaped and rod-shaped molecules [66].

An interesting question that emerges from this work is related to the behavior of the phase diagram in this kind of system by the addition of different inter- and intramolecular molecular interactions, for example, including dipolar and quadrupolar interactions or lateral chains attached to the backbone in such a way as to reproduce the properties of nonconventional discotic particles [36]. These issues will be addressed in future work.

\section{ACKNOWLEDGMENTS}

It is a great pleasure to thank H. H. Wensink, L. F. Rull, and J. M. Romero-Enrique for stimulating discussions and an anonymous referee for interesting comments and suggestions which allowed us to improve this work. Financial support for this work was been given by the U.K. Engineering and Physical Sciences Research Council (EPSRC), Grant No. EP/E016340, "Molecular System Engineering."
[1] L. Onsager, Ann. N.Y. Acad. Sci. 51, 627 (1949).

[2] A. Stroobants, H. N. W. Lekkerkerker, and D. Frenkel, Phys. Rev. Lett. 57, 1452 (1986).

[3] A. Stroobants, H. N. W. Lekkerkerker, and D. Frenkel, Phys. Rev. A 36, 2929 (1987).

[4] D. Frenkel, J. Phys. Chem. 92, 3280 (1988).

[5] D. Frenkel, H. N. W. Lekkerkerker, and A. Stroobants, Nature (London) 332, 822 (1988).

[6] J. A. C. Veerman and D. Frenkel, Phys. Rev. A 41, 3237 (1990).

[7] J. A. C. Veerman and D. Frenkel, Phys. Rev. A 43, 4334 (1991).

[8] S. C. McGrother, D. C. Williamson, and G. Jackson, J. Chem. Phys. 104, 6755 (1996).

[9] P. Bolhuis and D. Frenkel, J. Chem. Phys. 106, 666 (1997).
[10] J. D. Parsons, Phys. Rev. A 19, 1225 (1979).

[11] S. D. Lee, J. Chem. Phys. 87, 4972 (1987).

[12] S. D. Lee, J. Chem. Phys. 89, 7036 (1988).

[13] T. Odijk, Macromolecules 19, 2313 (1986).

[14] G. J. Vroege and H. N. W. Lekkerkerker, Rep. Prog. Phys. 55, 1241 (1992).

[15] M. Franco-Melgar, A. J. Haslam, and G. Jackson, Mol. Phys. 106, 649 (2008).

[16] P. T. Collings and M. Hird, Introduction to Liquid Crystals (Taylor \& Francis, London, 1997).

[17] C. Tschierske, Annu. Rep. Prog. Chem., Sect. C.: Phys. Chem. 97, 191 (2001).

[18] R. J. Bushby and O. R. Lozman, Curr. Opin. Colloid Interface Sci. 7, 343 (2002).

[19] S. Kumar, Chem. Soc. Rev. 35, 83 (2006). 
[20] S. Chandrasekhar, B. K. Sadashiva, and K. A. Suresh, Pramana 9, 471 (1977).

[21] D. Frenkel and R. Eppenga, Phys. Rev. Lett. 49, 1089 (1982).

[22] J. A. C. Veerman and D. Frenkel, Phys. Rev. A 45, 5632 (1992).

[23] M. A. Bates, J. Chem. Phys. 111, 1732 (1999).

[24] F. M. van der Kooij and H. Lekkerkerker, J. Phys. Chem. B 102, 7829 (1998).

[25] F. M. van der Kooij, K. Kassapidou, and H. N. W. Lekkerkerker, Nature (London) 406, 868 (2000).

[26] F. M. van der Kooij and H. N. W. Lekkerkerker, Philos. Trans. R. Soc. London, Ser. A 359, 985 (2001).

[27] M. A. Bates and D. Frenkel, Phys. Rev. E 57, 4824 (1998).

[28] R. Blaak, D. Frenkel, and B. Mulder, J. Chem. Phys. 110, 11652 (1999).

[29] B. S. John, C. Juhlin, and F. A. Escobedo, J. Chem. Phys. 128, 044909 (2008).

[30] A. Cuetos and B. Martinez-Haya, J. Chem. Phys. 129, 214706 (2008).

[31] J. Vieillard-Baron, J. Chem. Phys. 56, 4729 (1972).

[32] D. Frenkel, B. M. Mulder, and J. P. McTague, Phys. Rev. Lett. 52, 287 (1984).

[33] M. Allen and M. Wilson, J. Comput.-Aided Mol. Des. 3, 335 (1989).

[34] M. A. Bates and G. R. Luckhurst, J. Chem. Phys. 104, 6696 (1996).

[35] S. Höger, J. Polym. Sci., Part A: Polym. Chem. 37, 2685 (1999).

[36] S. Höger, V. Enkelmann, K. Bonrad, and C. Tschierske, Angew. Chem., Int. Ed. 39, 2267 (2000).

[37] J. S. Moore and J. Zhang, Angew. Chem., Int. Ed. Engl. 31, 922 (1992).

[38] D. Bong, T. Clark, J. Granja, and M. Ghadiri, Angew. Chem., Int. Ed. 40, 988 (2001).

[39] S. Höger, Chemistry (Weinheim, Ger.) 10, 1320 (2004).

[40] T. Kato, N. Mizoshita, and K. Kishimoto, Angew. Chem., Int. Ed. 45, 38 (2006).

[41] D. H. Zhao and J. S. Moore, Chem. Commun. (Cambridge) 2003807.

[42] S. Hoger, X. Cheng, A. Ramminger, V. Enkelmann, A. Rapp, M. Mondeshki, and I. Schnell, Angew. Chem., Int. Ed. 44, 2801 (2005).

[43] K. Nakao, M. Nishimura, T. Tamachi, Y. Kuwatani, H. Miyasaka, T. Nishinaga, and M. Iyoda, J. Am. Chem. Soc. 128,
16740 (2006).

[44] M. Iyoda, C. R. Chim. 12, 395 (2009).

[45] B. J. Lemaire, P. Davidson, J. Ferre, J. P. Jamet, D. Petermann, P. Panine, I. Dozov, and J. P. Jolivet, Eur. Phys. J. E 13, 291 (2004).

[46] B. J. Lemaire, P. Davidson, D. Petermann, P. Panine, I. Dozov, D. Stoenescu, and J. P. Jolivet, Eur. Phys. J. E 13, 309 (2004).

[47] B. J. Lemaire, P. Davidson, P. Panine, and J. P. Jolivet, Phys. Rev. Lett. 93, 267801 (2004).

[48] H. H. Wensink and G. J. Vroege, Phys. Rev. E 72, 031708 (2005).

[49] D. van der Beek, P. Davidson, H. H. Wensink, G. J. Vroege, and H. N. W. Lekkerkerker, Phys. Rev. E 77, 031708 (2008).

[50] J. D. Weeks, D. Chandler, and H. C. Andersen, J. Chem. Phys. 54, 5237 (1971).

[51] W. G. Hoover, Phys. Rev. A 31, 1695 (1985).

[52] S. Melchionna, G. Ciccotti, and B. L. Holian, Mol. Phys. 78, 533 (1993).

[53] J. Gao and J. H. Weiner, J. Chem. Phys. 91, 3168 (1989).

[54] D. M. Heyes and H. Okumura, Mol. Simul. 32, 45 (2006).

[55] W. Smith, Mol. Simul. 32, 933 (2006).

[56] W. Smith, I. T. Forester, and T. R. Todorov, The DL_POLY_2 user manual, CCLRC, Daresbury Laboratory (Daresbury, Warrington, U.K., 2009).

[57] M. P. Allen, G. T. Evans, D. Frenkel, and B. M. Mulder, Adv. Chem. Phys. 86, 1 (1993).

[58] R. J. Low, Eur. J. Phys. 23, 111 (2002).

[59] S. Singh, Phys. Rep. 324, 107 (2000).

[60] R. E. Webster, N. J. Mottram, and D. J. Cleaver, Phys. Rev. E 68, 021706 (2003).

[61] A. J. Crane, F. J. Martinez-Veracoechea, F. A. Escobedo, and E. A. Müller, Soft Matter 4, 1820 (2008).

[62] W. H. Press, S. A. Teukolsky, and B. P. Vetterking, Numerical Recipes in Fortran, 1st ed. (Cambridge University Press, London, 1986).

[63] A. G. Vanakaras and D. J. Photinos, Chem. Phys. Lett. 341, 129 (2001).

[64] M. P. Allen and D. J. Tildesley, Computer Simulation of Liquids (Oxford University Press, Oxford, 1987).

[65] D. Frenkel and B. Smit, Understanding Molecular Simulation, 2nd ed. (Academic Press, London, 2002).

[66] A. Godt, S. Duda, O. Ünsal, J. Thiel, A. Härter, M. Roos, C. Tschierske, and S. Diele, Chemistry (Weinheim, Ger.) 8, 5094 (2002). 